\title{
Inflated Egos of Chinese Undergraduates: A Cross-temporal Meta-analysis, 2005-2012
}

\author{
Xiaolan Fan, Jinfu Zhang \\ Southwest University, Chongqing, China
}

\begin{abstract}
A cross-temporal meta-analysis was applied to examine the changes in Chinese college students' narcissism level from 2005 to 2012. We collected the literature investigating college students by Zheng Yong's Narcissistic Personality Questionnaire in the past eight years. The results showed that, mean narcissism score was significantly correlated with year of data when weighted by sample size, suggesting that narcissism level of Chinese college students has been slowly increasing from 2005 to 2012; the overt narcissism level had no significant gender difference, while male college students had higher than female in covert narcissism.
\end{abstract}

Keywords: college students, overt narcissism, covert narcissism, cross-temporal meta-analysis, meta-analysis

\section{Introduction}

With the development of reform and opening-up, the politics, economy, culture and other fields in China have made great progress and our national conditions have completely changed. People's values, ethics, and the moral views gradually are also changing. Western culture has made its entry which impacts the golden mean of Chinese people under the background of collectivism culture. People on the basis of the original stressing collective cooperation, gradually begin to pursue personal value (Yan, 2011). Many bestsellers attract the attention of readers in the eye-catching signs such as, "how to show yourself", "you are the most important". Especially young adults who live in the Internet age can express different views and upload attractive photos snapped of themselves through social networks, mobile phones and other tools, anytime and anywhere. They grow up amid their family's voice of praise because most of them are the only child. Post-90s and post-00s generation is the main force of this group, described as self-centered, arrogant, disrespectful, but fragile. In November 2006, one survey done in Shanghai Songjiang district university town found that nearly a quarter of the students admitted to be very narcissistic, nearly a quarter of the students thought there was too much narcissist around, nearly $20 \%$ of the students had been attacked for being too narcissistic, in nearly 400 students $(\mathrm{Wu}, 2006)$. Narcissism as a kind of common personality trait has not brought to the attention of the Chinese people for a long time. It was considered as a negative trait, but we cannot treat it as the same situation. Because former studies in the west have found that narcissism is universal in normal population and moderate narcissism had positive effect to mental health.

In this study, we focus on subclinical narcissism personality, namely normal narcissism. In the non-clinical level, it is characterized foremost by a very positive and boastful view of self. Narcissism is

Xiaolan Fan, M.S. Student, Faculty of Psychology, Southwest University.

Jinfu Zhang, professor, Faculty of Psychology, Southwest University. Email: zhangif@swu.edu.cn 
generally believed as a long-term, diverse, comprehensive personality structure. It is individuals who fuss over and indulge in themselves, their own imagination and their image in the eyes of others. Narcissists think they are more superior to others, have a feeling of exaggerated self-importance, and believe themselves as entitled. Therefore, narcissism includes two meanings: On one hand, it is to love the self, on the other hand, it is to focus on the self.

The structure of narcissism is currently accepted by most psychologists that narcissism has two forms (Rose, 2002), which is put forward by Wink (1991). Narcissism can be divided into overt narcissism and covert narcissism. Overt narcissists have very direct expression, and always need the attention and admiration of others. They display superiority, and show interest in appreciation of others', success and themselves. Overt narcissists reflect their feeling of inflated self directly: strong expressive desire, superiority, too need to be the envy of others, entitled, and generally is independent, outgoing, optimistic and confident. The covert narcissists have potential inferiority, and always feel inferior to others, characterized by a lack of self-confidence and initiative, lack of interest in work and study. They are overly sensitive, anxious, insecure, but they will still self exaggerate self unconsciously.

Studies of narcissism in China were discussed almost from the perspective of literature, sociology, compared with the substantial researches in the west. Zheng Yong and Huang Li (2006) suggested narcissism could be divided into overt and covert narcissism; overt narcissism has four dimensions: authority, superiority, entitlement and self-admiration, and covert narcissism has three dimensions: vulnerability, entitlement and self-admiration in the context of Chinese culture. Henceforth, more studies of narcissism were presented, and some scholars had analyzed narcissistic traits' differences between such as gender and subject, but the results were not the same. It was concluded that college students' overt narcissism and covert narcissism both have no significant gender differences in Huang Li's (2004) master thesis. One study found that covert narcissism showed a significant difference in gender, and males were significantly higher than female (Linghu \& Shi, 2011). However, another study reported there was significant gender difference in the covert narcissism, and it was females who were significantly higher than males (LV, 2012).

For the divergence, meta-analysis is more effective and common practice to deal with. Meta-analysis was put forward by Glass formally, which aimed at discoveries existing and integrated numerous single results (Glass, 1976). It could derive a general conclusion from quantitative re-analyzing many literatures. Meta-analysis methods applied at present mostly were to compute the effect size. The commonly used statistical index is d, whose calculation method was put forward by Cohen, and Glass improved it. The formula id (Zhou, 1992): $d=(M e-M c) / S D, M e$ as experimental average, $M c$ as control group average (scale of norm score can also be regarded as control group score usually), $S D$ as the common standard deviation of the two groups. $S D$ 's calculation method current is as follows: $S D=\sqrt{\left(n_{e}-1\right) s_{e}^{2}+\left(n_{c}-1\right) s_{c}^{2} /\left(n_{e}+n_{c}-2\right)}$.

Meta-analysis technology has many advantages, as a kind of quantification method of literature review; it can integrate previous research results and get general conclusions without the original data. In recent years, its influence and application have been looming larger in the field of psychology and education, but many researchers suggest meta-analysis's limitation limits its application. For example, it depends too much on published results of study; the integration of research results may ignore differences in particular study; results of meta-analysis are susceptible to the quality and quantity of literature; effect size's calculation should consider the factors such as sampling, measuring error (Xia, 2005). 
In addition, Xin Ziqiang and his colleagues pointed out that general meta-analysis often cannot effectively deal with cohort effect-Many meta-analysis studies have found that the years of data collection were related to the research results, but they did not delve into this phenomeno (Xin \& Chi, 2008; Z. Xin, S. Xin, \& Zhang, 2011; Xin \& Zhang, 2009). For example, a study found that high school students' SCL-90 score had been increasing with the years and cohort effect accounted for $19.7 \%$ of the effect size, but no specific analysis for it (Huiyong \& Jinfu, 2006). Therefore, some researchers think that cohort effect would confuse results of meta-analysis, which should be controlled.

To solve the cohort effect in meta-analysis, professor Jean M. Twenge of the University of San Diego presented a special meta-analysis technology — cross-temporal meta-analysis, can be called cross-temporal study. It applies cross-sectional study design to discuss the differences or variances of large span time and age in meta-analysis. The design here is not as advance as cross-sectional study of individual development to construct method, but ratification, in other words, it connects each research in chronological order, so as to make those existing studies become transect sampling which coordinates historical development. The method as a special kind of meta-analysis is unique because the time is used as a bridge to connect psychological strictures with social indicators to discover factors of social change behind cohort effect. Twenge had studied some psychological measures with this method (Twenge, 2001a; 2001b), and used the traditional "birth cohort effect" to describe the phenomenon. Born group is not only on behalf of the individual's date of birth, but more important is that it shows the influences of decided specific social and cultural environment for individual growth.

Twenge suggested that it was a modified meta-analysis technique, also could be called within-scale meta-analysis. Cross-temporal meta-analysis does not compute an effect size for each study but instead examines the change in mean scores on psychological measures over time. Because the data are measured within one scale, it is possible to compare means across samples collected at different times directly. So, we could examine the correlation (weighted for sample size) between the mean scores and their years of data collection.

In this article, we examine change over time in narcissism, and the gender difference of overt and covert narcissism in college students.

\section{Method}

\section{Narcissistic Personality Questionnaire}

In China, the narcissistic personality questionnaire developed by Zheng Yong and Huang Li is the most popular instrument to measure narcissism, including overt and covert narcissistic personality subscales. There are four dimensions for overt narcissism: authority, superiority, entitlement and self-admiration, a total of 20 items; three dimensions for covert narcissism: vulnerability, entitlement and self-admiration, a total of 15 items. Authority reflects individuals' tendency of authority and leadership; Superiority reflects that individuals believe themselves as good, and are appreciated by others; Entitlement reflects that individuals think they are qualified and should get more from others in a way of utilization and exploitation; Self-admiration reflects the narcissists' an obvious self-focus and admire; Vulnerability reflects the characteristic of fragile and sensitive. Two subscales contain seven repeat items, so we get 28 -item formal narcissistic personality questionnaire. It is Likert 5-point scale, and the higher score, the more obvious narcissistic personality.

The two subscales' internal consistency coefficients are all above 0.7 , and split-half reliability coefficients are over 0.6 , which indicates that the questionnaire has good reliability; the items' compilation and dimension 
structure are based on well-known related scale, open questionnaire investigation, literature review and individual interview, so the scale has good content validity.

\section{Inclusion Rules}

Possible studies for the analysis were included or excluded on the basis of specific rules. To be included in this mate-analysis, a study had to meet the following criteria: (1) Research should use the same measurement tools, namely Zheng Yong and Huang Li narcissistic personality questionnaire; (2) Participants were Chinese students from university, not junior colleges or vocational schools, a two or three years colleges; (3) The research should report or we can get through contacting the author, the mean, standard deviation, sample size and other basic information, and cannot choose the report according to the narcissism score only the means of the high and low groups, etc.; and (4) The same data were used in different papers, we can only choose one.

In addition, we send mails to the authors to confirm the time of data collection and single-sex means, etc.. When the exact year was not available, according to the actual situation of domestic as well as the previous meta-analysis researches, the year of data collection was coded as two years prior to publication (Oliver \& Hyde, 1993; Xin \& Zhang, 2009).

\section{Literature Search and Variables' Coding}

These studies were located by searching three of the most important Chinese academic literature databases, CNKI, Weipu, and Wanfang. These databases together cover nearly all of the Chinese journals for the social sciences and a large number of doctoral dissertations and master's theses. So we used fields like "college students", "narcissistic personality", "overt narcissism", and "covert narcissism" to explore literature in the title, keywords and abstract. And according to the documents record of Zheng Yong and Huang Li's paper published in Psychological Science in 2005- "Overt and covert narcissism: A psychological exploration of narcissistic personality", we select from the retrieved literature up to the standard of the literature. Finally, we collected nine studies, involving 2,646 students from 2005 to 2013. According to the previous studies, we sorted included studies by time of data collection (seen as a continuous variable), gender ( $1=$ boys, $2=$ girls), journal type $(1=$ core journals, $2=$ general journals, $3=$ degree papers or conference proceedings $)$.

\section{Results}

\section{Correlations Between Mean Scores of Narcissism and Year}

To descript intuitively college students' narcissism level change trend along with the change of year since 2005, we used years as abscissa, narcissism scores as ordinate to draw a scatter diagram (see Figure 1). From the scatter plot, narcissistic level is roughly linear growth trend, and university students' overall level of narcissism is gradually rising along with the years.

In the subsequent correlation analysis, years are significantly associated with the college students' level of narcissism (not control sample correlation coefficient $r=0.90$, after controlling $r=0.89, p<0.01$ ). This shows that from 2005 to 2012, Chinese college students' level of narcissism is on the rise trend.

Compared with normal meta-analysis, results of cross-temporal meta-analysis will be affected by the literature or the characteristics of the sample. A meta analysis for nearly 10 years in high school students SCL-90 survey did by Fan Huiyong, Zhang Jinfu, found that the literature quality effect accounted $26.6 \%$ for the effect of the variation factors. In this study, the literature source may be an important variable, but because 
the standard samples we can get are too little, they cannot be subdivided and compared, so this work can be done deeper in the future.

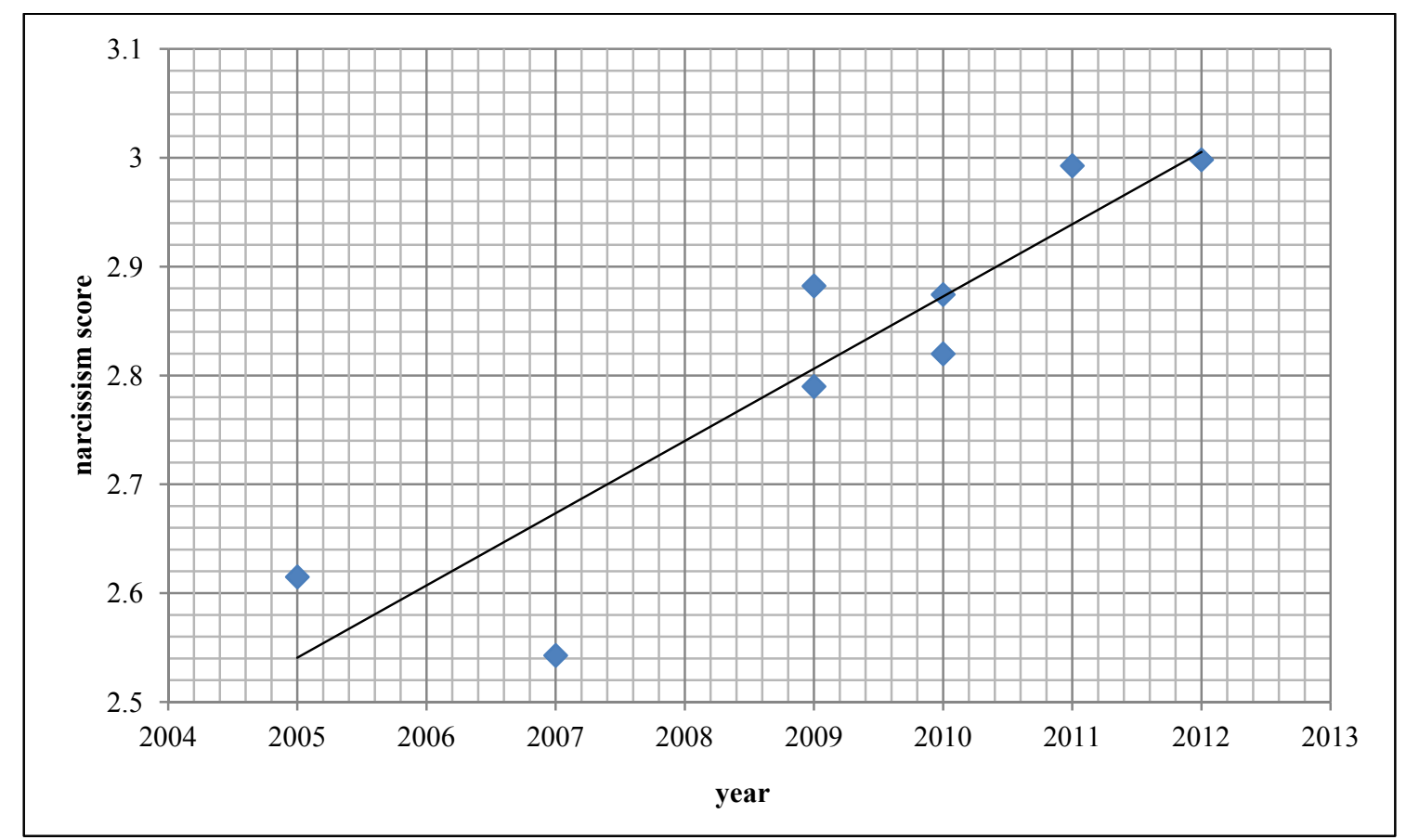

Figure 1. Changes in Chinese students' narcissism, 2005-2012.

The above results showed that Chinese college students' narcissism increased but we can hardly know how much narcissism increased from these results. To find the magnitude of narcissism change, we used regression equations to predict the mean scores of the first and last year of included studies. With controls, the mean score increased from 1.83 in 2005 to 2.29 in 2012. Thus, it rose $0.46,0.86$ standards ( $S D=0.54$ ) from 2005 to 2012. According to Cohen (1977), this was a big effect size $(d>0.8)$. We converted the $d$ value $(0.86)$ into variance explained by year and found the proportion was $79 \%$.

Table 1

Change Sizes of Narcissism

\begin{tabular}{llllll}
\hline$M 2005$ & $M 2012$ & $M$-change & $S D$ & $d$ & $R$-Square \\
\hline 1.83 & 2.29 & 0.46 & 0.54 & 0.86 & 0.79 \\
\hline
\end{tabular}

\section{Gender Differences of Overt and Covert Narcissism}

To discuss college students' gender differences of overt and covert narcissism, we did meta-analysis on those researches which have provided male and female college students' overt and covert narcissism mean scores and standard deviations. Firstly, we calculated the gender effect sizes for each study, then according to the weight to calculate the average effect sizes. In terms of all the documents that we can collect, gender's average effect size on overt narcissism is 0.18 which was after weighting, and 0.25 for covert narcissistic personality. The results are shown in Table 2.

Effect size can be explained from multiple perspectives, and here we are based on Cohen experience standard directly. He divided the effect sizes (absolute value) into small ones $(d=0.2)$, medium ones $(d=0.5)$ 
and big ones $(d=0.8)$. So, in this study the effect of gender on the overt narcissism was small effect size, suggesting that male and female college students' overt narcissism mean difference was not significant; the gender effect on covert narcissism was nearly medium effect, which showed that $\mathrm{m}$ boys' covert narcissism was higher than girls'.

Table 2

College Students' Overt Covert Narcissism Mean Effect on Gender

\begin{tabular}{llll}
\hline Style & $d$ & $S D$ & $S E$ \\
\hline Overt & 0.18 & 0.073 & 0.037 \\
Covert & 0.25 & 0.078 & 0.039 \\
\hline
\end{tabular}

\section{Discussion}

\section{College Students' Narcissism Rising Over the Past Eight Years}

This study applied cross-temporal meta-analysis to examine university students' narcissism cohort effect, found that students narcissism cohort effect significantly. That is to say, from 2005 to 2012, Chinese university students' narcissism had been rising with the progress of the age, increased by 0.86 standard deviations in eight years. The result above was consistent with the result of western college students NPI, which found by Twenge that American college students' narcissism was significantly related to years between 1979 and 2006, and NPI scores have increased 0.33 standard deviations from 1982; now two-thirds of American college students' narcissism score is higher than the score of 1979-1985, up 30\% (Twenge, Konrath, Foster, Keith Campbell, \& Bushman, 2008).

Since this study was based on Huang Li and Zheng Yong's narcissistic personality questionnaire which developed in 2005, though researched applied the questionnaire are on the rise, but up to now studies can be included in are few, and time across is shorter than other across-temporal researches, only eight years. Therefore, we can't rule out the possibility of accidental factors in the cohort effect of this period. That is to say, the stability of the results need includes more available literature to verify and improve. But, we still can see the influence of cohort effect on college students' psychological characteristics from the thin evidence. This also agrees with the previous cross-temporal researches about college students' mental health, anxiety and other psychological characteristics, and all can suggest that the macroscopical social environment influences on college students. It is easy to speculate that between 2005 and 2012, college students mainly from after 80s to after 90s, and China's one-child policy at this time also has the obvious effect. Most of both urban and rural families are only child, and after 90s college students tend to be the center of the family, grew up in the elder's care and appreciation, and tend to be self-centered, enjoying the privileges, etc.. Combined with the development of social economy and culture, below edification of autolatry in western culture, as a whole, these young people would pursue more self-expression and self-affirmation.

\section{College Students' Gender Difference of Overt and Covert Narcissism}

After meta-analysis, we found that the average effect of overt narcissism was small size, means that there was no significant difference in the overall male and female college students' overt narcissism. While male and female college students' covert narcissism was of difference because of the nearly medium effect size. Although some previous studies found no gender differences, but also found that overt and covert narcissism were gender differences. Our study was based on relatively large samples, the conclusion should be more 
general, and more reliable, but it does not directly deny the conclusion of gender differences in specific samples.

Since papers we collected did not report male and female's correlation coefficients of overt and covert narcissism respectively, or their standard deviations, we cannot produce meta-analysis for male and female's each total narcissism, but we still found they were both on a rising trend in two dimensions.

\section{Limitations}

Although we obtained more valuable results above, but there are still some limitations in this study. First, in terms of measurement tool, what study chose is only Huang Li and Zheng Yong's narcissistic personality questionnaire. However, there are other tools that are used, such as western NPI, HSNS and questionnaires developed by domestic researchers. In the future, study can be made with scales above to verify our results.

Secondly, in terms of samples, the scale is relatively new, so the number of available papers is limited. We regret that we cannot find Huang Li's earliest data used in her graduation thesis in 2004, losing a set of important data in this study. Especially when considering cross sectional meta-analysis, we can't really get samples across the times, so the tested cohort effect still remains to continue to certify.

\section{References}

Cohen, J. (1977). Statistical power analysis for the behavioral sciences. San Diego, C.A.: Acanemic Press.

Gao, Z., Wang, R., Chang, Y., Yan, N., \& Meng, G. (2013). Age characteristics of narcissism and influence of parents upbringing. China Journal of Health Psychology, 21(4), 603-605.

Glass, G. V. (1976). Primary, secondary, and meta-analysis of research. Educational Researcher, 3-8.

Huang, L. (2004). The research on undergraduates' narcissistic personality and its relationships with psychological well-being. (Mater's thesis, Southwest Nornal University, Chongqing, China).

Huang, Y. (2011). High school students narcissistic personality, achievement motivation and self-handicapping (Mater's thesis, East China Normal University, Shanghai, China).

Huiyong, F., \& Jinfu, Z. (2006). A Meta-analysis of reports concerning SCL-90 and Chinese middle school students during the past 10 years. Psychological Science, 28(6), 1424-1426.

Jiang, Y. (2007). Covert and overt narcissists' attack behavior differences under the company (Master's thesis, Southwest University, Chongqing, China).

Linghu, M., \& Shi, C. (2011). The relationship of college students narcissism and achievement motivation. China Journal of Health Psychology, 19(4), 454-456.

LV, Y. (2012). College students narcissism, self-esteem and achievement motivation (Master's thesis, Tianjin Normal University, Tianjin, China).

Oliver, M. B., \& Hyde, J. S. (1993). Gender differences in sexuality: A meta-analysis. Psychological Bulletin, 114(1), 29.

Rose, P. (2002). The happy and unhappy faces of narcissism. Personality and Individual Differences, 33(3), 379-391.

Twenge, J. M. (2001a). Birth cohort changes in extraversion: A cross-temporal meta-analysis, 1966-1993. Personality and Individual Differences, 30(5), 735-748.

Twenge, J. M. (2001b). Changes in women's assertiveness in response to status and roles: Across-temporal meta-analysis, 1931-1993. Journal of Personality and Social Psychology, 81(1), 133-145.

Twenge, J. M., Konrath, S., Foster, J. D., Keith Campbell, W., \& Bushman, B. J. (2008). Egos inflating over time: A cross-temporal meta-analysis of the narcissistic personality inventory. Journal of Personality, 76(4), 875-902.

Wink, P. (1991). Two faces of narcissism. Journal of Personality and Social Psychology, 61(4), 590.

Wu, X. (2006). Shanghai Youth Daily. Retrieved from http://www.chinanews.com/edu/news/2006/11-22/825086.shtml

Xia, L. (2005). On some basic problems of meta-analysis method. Journal of Shanxi Teachers University (Social Science Edition), 32(3), 34-38.

Xin, Z., \& Chi, L. (2008). Cross-temporal: Psychological development in social changes with meta-analysis. Journal of East China Normal University (Educational Sciences), 26(2), 44-51. 
Xin, Z., Xin, S., \& Zhang, M. (2011). Changes in college students' anxiety level in 1993-2009: A cross-temporal meta-analysis. Psychological Development and Education, 27(6), 648-653.

Xin, Z., \& Zhang, M. (2009). Changes in Chinese middle school students' mental health (1992-2005): A cross-temporal meta-analysis. Acta Psychologica Sinica, 41(1), 69-78.

Yan, K. (2011). The comparative study of Chinese and American college students narcissistic personality and cognitive decision-making (Master's thesis, Shanxi Normal University, Xian, Shaanxi,China).

Zhang, B. (2012). College students' parents upbringing, narcissistic personality and offensive (Master's thesis, Hangzhou Normal University).

Zhang, S. (2011). Study on the relationship between college students narcissism and satisfaction of relationship. Science Tribune, $28,221-222$.

Zhao, J., \& Zhang, H. (2007). Narcissism research progress. Journal of Psychiatry, 20(1), 52-57.

Zheng, Y., \& Huang, L. (2006). Overt and covert narcissism: A psychological exploration of narcissistic personality. Psychological Science, 28(5), 1259-1262.

Zhou, L. (1992). Introduce of meta-analysis. Psychological Development and Education, 8(2), 27-31. 